\title{
Time of interferon- $\beta$ 1a injection and duration of treatment affect clinical side effects and acute changes of plasma hormone and cytokine levels in multiple sclerosis patients
}

\author{
T Kümpfel ${ }^{1}$, M Schwan ${ }^{2}$, Th Pollmächer ${ }^{4}$, A Yassouridis ${ }^{2}, M$ Uhr $^{2}$, C Trenkwalder $^{3}$ \\ and $F$ Weber ${ }^{2}$
}

\begin{abstract}
During initiation of interferon-beta (IFN- $\beta$ ) therapy, many multiple sclerosis (MS) patients experience systemic side effects which may depend on the time point of IFN- $\beta$ injection. We investigated the time course of plasma hormone-, cytokine- and cytokine-receptor concentrations after the first injection of IFN- $\beta$ either at 8.00 a.m. (group A) or at 6.00 p.m. (group B) and quantified clinical side effects within the first $9 \mathrm{~h}$ in 16 medication free patients with relapsing-remitting MS. This investigation was repeated after 6-month IFN- $\beta$ therapy.

Plasma ACTH and cortisol concentrations followed their physiological rhythms, with lower levels in the evening compared to the morning, but raised earlier and stronger in group B after IFN- $\beta$ administration. IFN- $\beta$ injection in the evening led to a prompter increase of plasma IL- 6 concentrations and temperature during the first hours and correlated to more intense clinical side effects compared to group A. Plasma IL-10 concentrations increased more in group A compared to group B, but sTNF-RI and sTNFRIl concentrations raised $7 \mathrm{~h}$ after IFN- $\beta$ injection only in group B. Acute effects on plasma hormone and cytokine concentrations adapted after 6-month IFN- $\beta$ treatment, while diurnal variations were still present. Baseline sTNF-RII concentrations were elevated after 6-month IFN- $\beta$ therapy only in group A.

Our results show that time point of IFN- $\beta$ injection has differential effects on acute changes of plasma hormone and cytokine concentrations and is related to systemic side effects. This may have implications on the tolerability and effectiveness of IFN- $\beta$ therapy. Multiple Sclerosis 2007; 13: 1138-1145. http://msj.sagepub.com
\end{abstract}

Key words: time point of injection; interferon- $\beta$; cytokine; hormones; side effects; multiple sclerosis

\section{Introduction}

Interferon- $\beta$ (IFN- $\beta$ ) is an established therapy for multiple sclerosis (MS), but in the initial phase of IFN- $\beta$ therapy patients often suffer from side effects such as fever, chills, headache and myalgias which may lead to discontinuation of therapy $[1-3]$. We and others recently showed that IFN- $\beta$ injection leads to immediate increases of plasma cytokine and hormone concentrations such as interleukin-6 (IL-6), tumour-necrosis-factor- $\alpha$ (TNF- $\alpha$ ), cortisol, adrenocorticotropin hormone (ACTH) and human growth hormone (hGH) within the first hours [4-6]. The inter-relation between these acute changes of plasma cytokine and hormone concentrations and the occurrence of clinical side effects after IFN- $\beta$ administration remains unclear and has not yet been quantified by in vivo studies. In addition, it is not known if time point of IFN- $\beta$ injection influences immunological and

\footnotetext{
${ }^{1}$ Institute of Clinical Neuroimmunology, Klinikum Großhadern, Ludwig-Maximilians-Universität, Munich, Germany

2 Max-Planck-Institute of Psychiatry, Sections of Neurology, Clinical Chemistry and Statistics, Munich, Germany

${ }^{3}$ Department of Clinical Neurophysiology, University of Goettingen, Germany

${ }^{4}$ Centre of Mental Health, Klinikum Ingolstadt, Ingoldstadt, Germany

Author for correspondence: Dr Tania Kümpfel, Clinical Institute of Neuroimmunology, Klinikum Großhadern, Marchioninistr. 15, 81377 München, Germany. E-mail: tania.kuempfel@med.uni-muenchen.de Received 13 November 2006; accepted 19 February 2007
} 
hormonal parameters as well as systemic side effects.

Circadian rhythms of endogenous plasma hormone concentrations, such as cortisol, can have an impact on immunological parameters and tolerability as well as on effectiveness of immunosuppressive drugs [7]. Principles of chronotherapy are already applied for several diseases such as cancer, coronary heart disease or arthritis, but might also play a role in immunomodulatory therapies such as IFN- $\beta$ [8-10].

As a first step we investigated the time course of plasma hormone-, cytokine- and cytokine receptor concentrations after the first injection of IFN- $\beta$ either at 8.00 a.m. (group A) or at 6.00 p.m. (group B) and quantified systemic side effects within the first $9 \mathrm{~h}$ in 16 medication free MS patients. All patients were re-evaluated with the same protocol after 6-month IFN- $\beta$ therapy.

\section{Patients and methods}

\section{Patients}

Sixteen untreated patients (five male, 11 female; age: $35 \pm 9$ years, expanded disability status scale [EDSS]: median: 1.5; range: 1-4) with relapsingremitting MS in a stable phase were investigated. All patients met the diagnostic criteria for multiple sclerosis (McDonald et al.) and fulfilled indication for treatment with IFN- $\beta$ [11]. No patient had received steroids during the last 6 weeks before study entry or any other immunomodulatory or immunosuppressive treatment prior to treatment with IFN- $\beta$. Laboratory blood tests and urine tests were performed to exclude acute infectious diseases. The study protocol was approved by the State Board of Physicians' ethics committee (Bayerische Landesärztekammer). All patients gave written informed consent.

\section{Experimental design}

Patients were investigated with the same protocol at the beginning of IFN- $\beta$ therapy $\left(22 \mu \mathrm{g}\right.$ Rebif ${ }^{\circledR}$ subcutaneously (s.c.), three times a week) and 6 months later after continuous IFN- $\beta$ treatment. Patients were randomly assigned in two groups with eight patients per group: Group A: IFN- $\beta$ injection in the morning at 8.00 a.m. Group B: IFN- $\beta$ injection in the evening at 6.00 p.m.

On every occasion an intravenous cannula was inserted into a cubital vein at 8.00 a.m. resp. 6.00 p.m. and kept patient by normal saline infusion Blood was taken every 60 min during the following $9 \mathrm{~h}$ through this line. After the first blood sample had been taken for baseline evaluation, patients received $22 \mu \mathrm{g}$ IFN- $\beta$ s.c. and were observed during the following $9 \mathrm{~h}$; blood pressure, heart rate and temperature were monitored hourly. Temperature was measured with a digital ear thermometer throughout the whole study period. Systemic side effects (chills, headache, muscle and joint pain) were documented by an independent study nurse, which observed and questioned all patients during the observation period. Classification of side effects was as follows: $0=$ no side effects, $1=$ mild side effects, $2=$ moderate side effects, $3=$ severe side effects.

\section{Determination of plasma cytokine and hormone concentrations}

Blood was collected into chilled tubes containing EDTA and aprotinin as a peptidase inhibitor and immediately centrifuged. Plasma was frozen and stored at $-70^{\circ} \mathrm{C}$. Serum levels of IL- 6 , TNF- $\alpha$, soluble tumour necrosis factor receptor I and II (sTNF-RI, sTNF-RII) and interleukin-10 (IL-10) were quantified using commercial enzyme linked immunosorbent assay (ELISA) kits (IL-6 high sensitivity and IL-10 both from R\&D Systems, Minneapolis; TNF- $\alpha$, sTNF-RI and sTNF-RII from Biosource Europe, Belgium) according to the instructions of the manufacturers. The lower limits of detection for IL-6, IL10, TNF- $\alpha$, sTNF-RI and sTNF-RII were $0.09 \mathrm{pg} / \mathrm{mL}$, $0.5 \mathrm{pg} / \mathrm{mL}, \quad 3 \mathrm{pg} / \mathrm{mL}, \quad 50 \mathrm{pg} / \mathrm{mL}$ and $100 \mathrm{pg} / \mathrm{mL}$, respectively.

Concentrations of IL- 6 and TNF- $\alpha$ were determined every hour from 8.00 a.m. to 5.00 p.m. or 6.00 p.m. to 3.00 a.m. and concentrations of sTNFRI and sTNF-RII as well as of IL-10 every $2-3 \mathrm{~h}$.

Hormone levels of cortisol, ACTH and hGH were also determined every hour. They were measured using commercial radioimmunoassays (RIA) or chemiluminescence immunoassays (cortisol: RIA, DRG Instruments, Germany; ACTH: RIA, hGH: Chemiluminiscence Immunoassay, all Nichols Institute Diagnostics, San Juan Capistrano, CA, USA). Inter- and intraassay variation was less than $10 \%$ for all hormones.

\section{Statistical analysis}

To reduce the necessary number of statistical tests and keep the corrected level of significance in acceptable limits, we divided the entire time interval into the following five subintervals in group A/B:

1) Baseline

2) First interval: 9.00 a.m. and 10.00 a.m./7.00 p.m. and 8.00 p.m. 
3) Second interval: 11.00 a.m., 12.00 a.m. and 1.00 p.m./9.00 p.m., 10.00 p.m. and 11.00 p.m.

4) Third interval: 2.00 p.m. and 3.00 p.m./00.00 p.m. and 01.00 a.m.

5) Fourth interval: 4.00 p.m. and 5.00 p.m./02.00 a.m. and 03.00 a.m.

For inferential statistics of the various variables (hormones and cytokines) two-factorial multivariate analyses of variance (MANOVA) with repeated measures design was performed. Thereby group was a between-subjects factor with two levels and time a within-subjects factor with two (corresponding to the beginning or end of the therapy) or five levels (according to the five subintervals). As nominal level of significance $\alpha=0.05$ was accepted and corrected according to the Bonferroni procedure for posteriori tests (tests with contrasts or univariate $F$-tests), in order to keep the type I error less or equal to 0.05. Results of cytokine and hormone concentrations are expressed as mean \pm standard error (SE) in the text and in all tables and figures.

Side effects were compared between groups using the non-parametric Mann-Whitney U-test. For the variables temperature, cortisol and IL-6, the maximum value (tmax, cortmax, IL-6max) and the difference between the maximum rise and the baseline value (tdmax, cortdmax, IL-6dmax) were determined. Correlation analysis was performed using Spearman-Rho.

\section{Results}

\section{Clinical side effects}

At the initiation of IFN- $\beta$ therapy systemic side effects tended to be more intense in group B than in group A $(1.2 \pm 0.2$ versus $2.1 \pm 0.3$; MannWhitney $U$-test, $P=0.052$ ) after IFN- $\beta$ injection. Side effects correlated positively with IL- 6 max $(r=0.82 ; P<0.001)$, but not with other variables (Table 1).

\section{Temperature, blood pressure and heart rate}

Figure 1 shows diurnal differences of temperature with higher baseline values in the evening. Treatment with IFN- $\beta$ induced a significant rise in temperature, which was in the second and third interval significant higher in group $B$ than in group A. Similar changes were observed for heart rate values, which raised in both groups but earlier in group B after IFN- $\beta$ injection (data not shown). Blood pressure was not affected by IFN- $\beta$ injection in both groups (data not shown). Mean changes of temperature correlated well with mean changes of IL-6 $(r=0.945, P<0.001)$, and heart rate $(r=0.889, P<0.001)$, but not with the other variables.

\section{Cytokine measurements}

IFN- $\beta$ treatment induced an acute rise of IL- 6 plasma concentrations which was at the beginning steeper in group $B$ and remained high throughout the whole observation period in both groups, but started to decrease again after 8-9 h (Figure 1). TNF- $\alpha$ values did not change after IFN$\beta$ injection during the $9 \mathrm{~h}$ observation period in both groups, but exhibited diurnal variations with higher mean plasma concentrations in group B (Figure 2). In contrast plasma IL-10 levels

Table 1 Injection time ( 1 = morning; 2 = evening) and side effects (rated from $0-3$ ) compared to the maximum rise in IL-6 and temperature in all patients at the beginning of therapy. IL-6 max correlated with side effects (Spearman-rho test, $P<0.001$ ). Side effects tend to be more intense in the evening (1.2 \pm 0.2 versus $2.1 \pm 0.3$; Mann-Whitney $U$-test, $P=0.052)$

\begin{tabular}{lllll}
\hline Patient & Injection time & Side effects & IL-6 max $(\mathrm{pg} / \mathrm{mL})$ & Temp. max $\left(\mathrm{C}^{\circ}\right)$ \\
\hline P001 & 1 & 1 & 4.9 & 37.6 \\
P002 & 1 & 1 & 8.6 & 38.0 \\
P004 & 1 & 2 & 12.9 & 38.7 \\
P006 & 1 & 2 & 10.2 & 38.4 \\
P009 & 1 & 0 & 9.3 & 37.8 \\
P010 & 1 & 1 & 4.6 & 37.4 \\
P012 & 1 & 2 & 10.2 & 37.7 \\
P015 & 1 & 1 & 27.0 & 38.2 \\
P003 & 2 & 3 & 5.2 & 38.0 \\
P005 & 2 & 2 & 11.0 & 37.4 \\
P007 & 2 & 2 & 12.4 & 38.6 \\
P008 & 2 & 2 & 12.4 & 38.6 \\
P011 & 1 & 16.5 & 38.3 \\
P013 & 2 & 3 & 8.8 & 38.8 \\
P014 & 2 & 3 & 21.0 & 39.0 \\
P016 & 2 & 27.0 & 38.0 \\
\hline
\end{tabular}



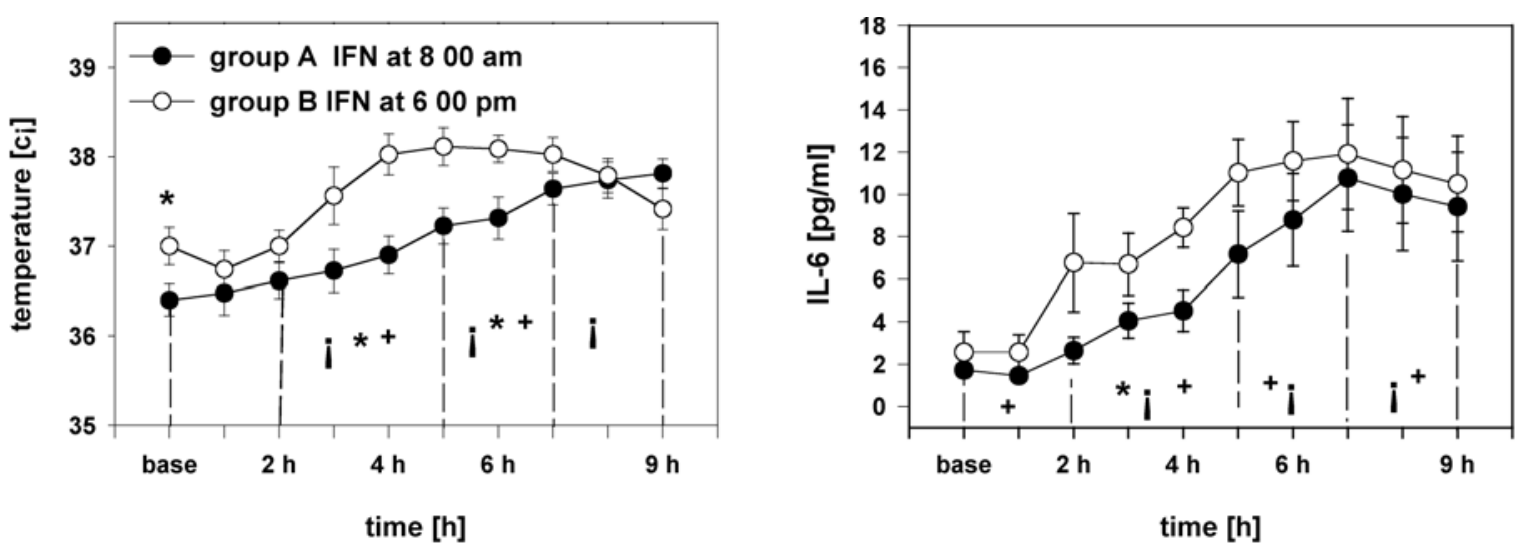

Figure 1 Time course of temperature and IL-6 in $16 \mathrm{MS}$ patients after administration of $22 \mu \mathrm{g}$ IFN s.c. either in the morning at 8.00 a.m. (group A) or in the evening at 6.00 p.m. (group B). The subdivision into different time intervals for statistical analysis is indicated in the figure by dotted lines. *Significant differences between group $A$ and $B,^{\circ}$ or + significantly higher compared to baseline in group $A$ or $B(P<0.05$, MANOVA). Values are mean \pm SE.
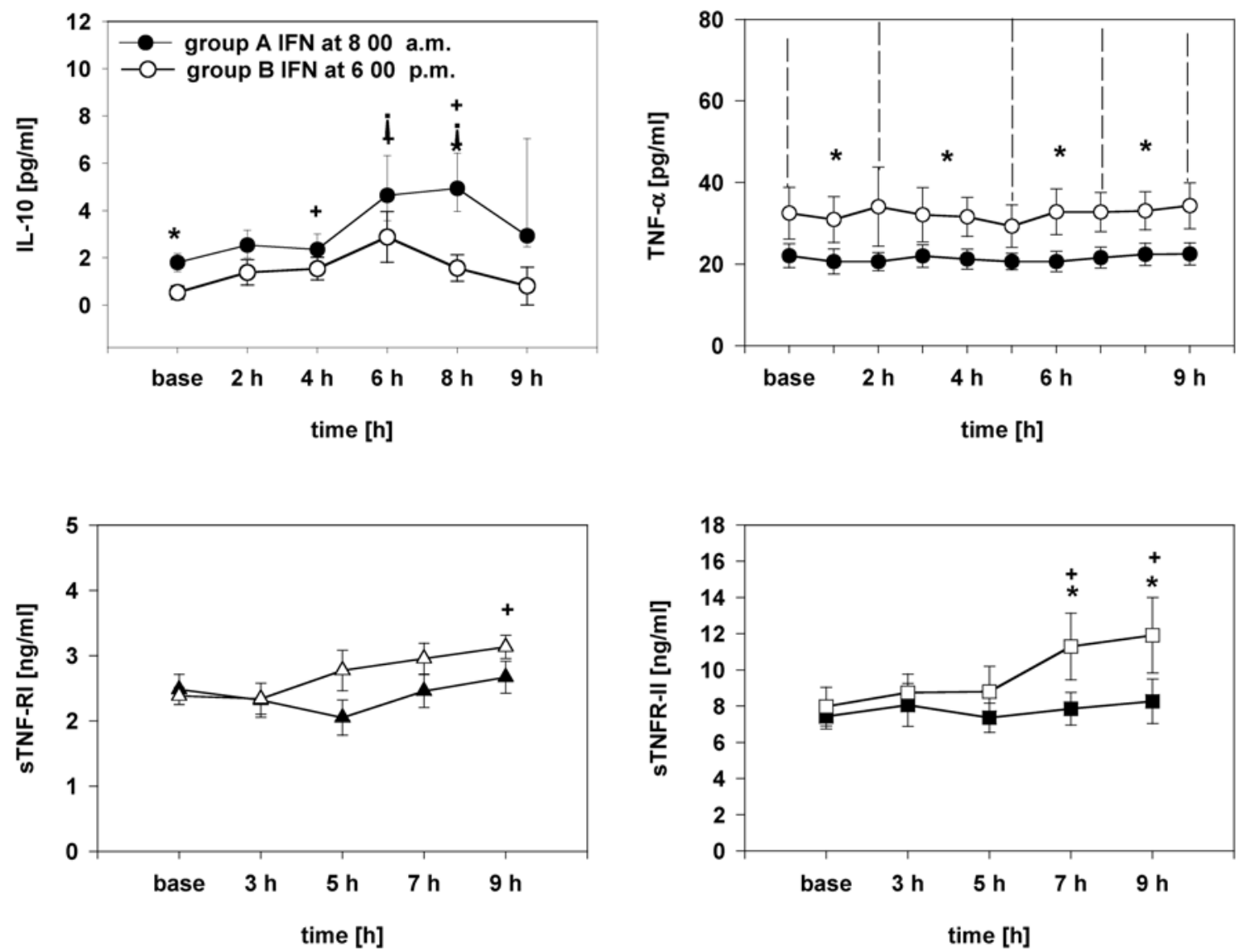

Figure 2 Time course of plasma IL-10, TNF- $\alpha$, sTNF-RI and sTNF-RII concentrations in 16 MS patients after administration of $22 \mu \mathrm{g}$ IFN s.c. either in the morning at 8.00 a.m. (group A) or in the evening at 6.00 p.m. (group B). The subdivision into different time intervals for statistical analysis is indicated in the figure for TNF- $\alpha$ by dotted lines. *Significant differences between group $A$ and $B,{ }^{\circ}$ or + significantly higher compared to baseline in group $A$ or $B(P<0.05$, MANOVA $)$. Values are mean $\pm S E$.

at baseline were higher in the morning compared to the evening. Towards the end of the observation period the increases of plasma IL-10 levels were significantly higher in group A compared to group B before decreasing again (Figure 2). Soluble cytokine receptors sTNF-RI and sTNF-RII raised $7 \mathrm{~h}$ to $9 \mathrm{~h}$ after IFN- $\beta$ injection only in group B (Figure 2). 


\section{Hormonal parameters}

Figure 3 reflects the endogenous diurnal rhythms of plasma cortisol concentrations with significant higher hormone concentrations in the morning group compared to the evening group at baseline. In both groups cortisol concentrations followed their physiological rhythms and decreased in the first $2 \mathrm{~h}$ after IFN- $\beta$ injection before they started to increase again. This increase was earlier in the evening compared to the morning group. ACTH concentrations showed similar results with an earlier and more pronounced increase of ACTH in the evening group. In both groups hGH plasma levels raised significantly $2-3 \mathrm{~h}$ after IFN- $\beta$ administration and dropped down towards baseline levels at the end of the observation period.

\section{Follow-up investigation after 6 months}

After 6-month IFN- $\beta$ therapy systemic side effects after injection disappeared or diminished in all

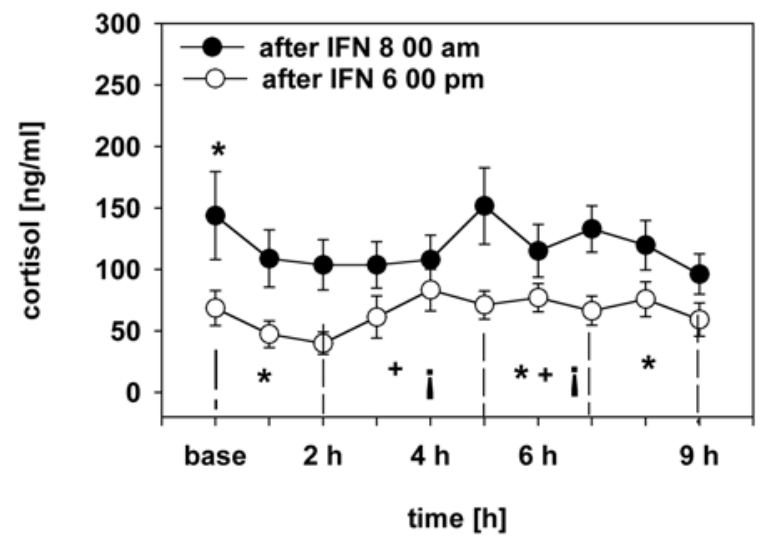

patients (data not shown). Besides diurnal variations, most acute changes after IFN- $\beta$ injection adapted after six month treatment with IFN- $\beta$. Increases of plasma IL- 6 after IFN- $\beta$ injection were diminished but still more pronounced in the evening group (data not shown).

Plasma IL-10 levels were higher in the morning group during the first $6 \mathrm{~h}$ in the follow up investigation. Baseline sTNF-RII values of the follow-up investigation in group A were significantly higher compared to baseline values of the initial investigation $(10.7 \pm 1.3 \mathrm{ng} / \mathrm{mL}$ versus $7.4 \pm 0.6 \mathrm{ng} / \mathrm{mL}$, data not shown).

\section{Discussion}

In the present study we injected IFN- $\beta$ at two different time points (morning versus evening) in order to investigate the influences of diurnal rhythms on plasma, the acute changes of hormone and cytokine concentrations after IFN- $\beta$ administration and its possible relation to systemic side

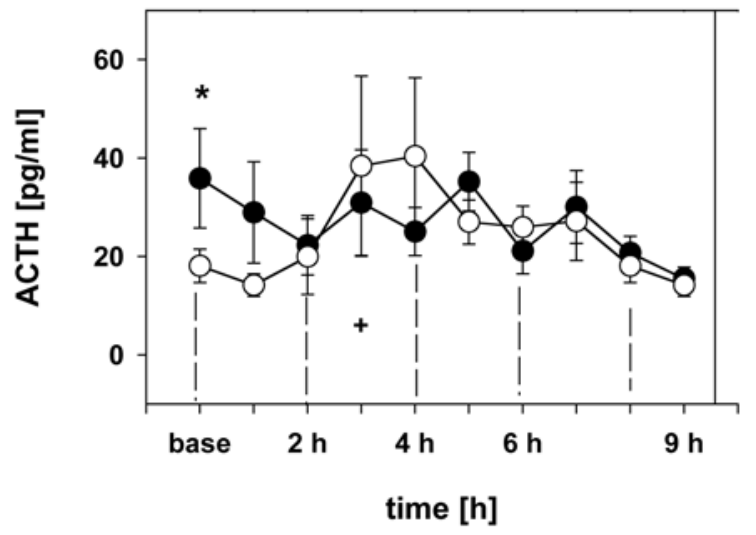

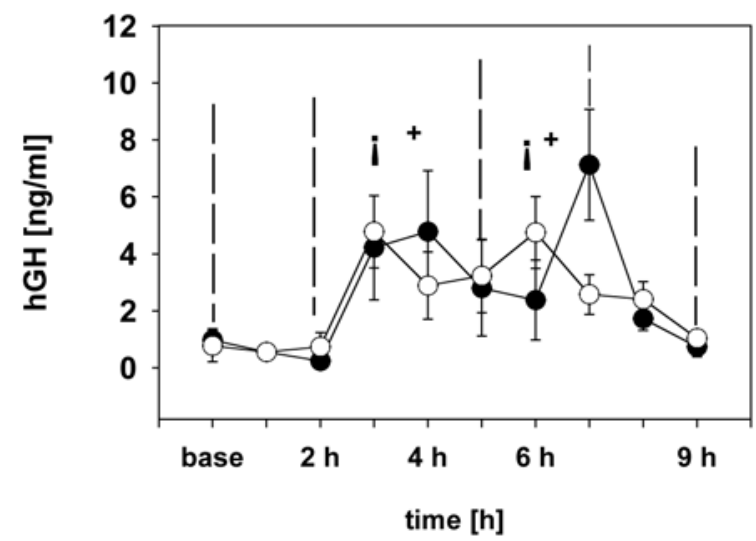

Figure 3 Time course of plasma cortisol, ACTH and hGH concentrations in $16 \mathrm{MS}$ patients after administration of $22 \mu \mathrm{g}$ IFN s.c. either in the morning at 8.00 a.m. (group A) or in the evening at 6.00 p.m. (group B). The subdivision into different time intervals for statistical analysis is indicated in the figures by the dotted lines. *Significant differences between group $A$ and $B$, ${ }^{\circ}$ or + significantly higher compared to baseline or first time interval in group $A$ or $B(P<0.05 ;$ MANOVA). Values are mean $\pm S E$. 
effects. Since we and others already demonstrated in earlier studies that treatment with placebo compared to IFN- $\beta$ injection did not lead to changes of plasma hormone and cytokine concentrations, a placebo treatment (eg, injection with saline) was not included in this study [4-6]. In addition, pretreatment with non-steroidal anti-inflammatory drugs (NSAIDs) was not allowed, because it is known that NSAID's reduce the immediate changes of plasma hormone and cytokine concentrations after IFN- $\beta$ injection and could therefore mask effects of circadian rhythms [5].

Our results clearly demonstrate that time point of IFN- $\beta$ injection affected acute changes of plasma hormone and cytokine concentrations as well as tolerability of IFN- $\beta$ administration in MS patients. We could show for the first time that systemic side effects correlated to the absolute rise of plasma IL6 concentrations. A role for IL- 6 in the mediation of flu-like symptoms (FLS) has already been suggested by former studies, which found that IL-6 increased 2-4h after IFN injection [4-6]. It is also supported by an observation of Montalban et al. who demonstrated that patients with fever have a higher percentage of IL- 6 producing peripheral blood mononuclear cells when IFN- $\beta$ therapy is initiated [12]. However, three patients in our study developed despite only modest increases of IL-6 (max: below $10 \mathrm{pg} / \mathrm{mL}$ ) a rise in temperature up to $38^{\circ} \mathrm{C}$ suggesting additional mechanisms of fever induction after IFN injections in these patients. Interleukin-1 (IL-1) is unlikely to be a possible inductor of fever, because IL-1 concentrations remained unchanged during the first hours after IFN injection in several studies [4-6]. In contrast, $\gamma$-interferon (IFN- $\gamma$ ) could be involved in the mediation of fever after IFN- $\beta$ injection since it was described to be enhanced shortly after IFN- $\beta$ injection and to induce side effects such as fever and chills $[13,14]$.

The rise of temperature and IL- 6 after IFN- $\beta$ administration was more pronounced during the first hours in the evening group which experienced also more systemic side effects than the morning group. This indicates that MS patients might be more susceptible to IFN- $\beta$ in the evening when physiological cortisol levels are low and body temperature is higher compared to the morning. It seems that the higher endogenous cortisol levels in the morning group suppressed a steep rise of IL-6 and temperature after IFN- $\beta$ during the first hours. An influence on the tolerability of drugs by circadian rhythms of cortisol has already been reported by Pollmächer et al., who also found a stronger increase of temperature in the evening after endotoxin administration when endogenous cortisol levels were low [15]. Circadian rhythm of body temperature, which generally reaches maximum values in the evening around 6.00 p.m. to 8.00 p.m. in healthy subjects, might have an additional impact on the temperature rise in the evening group, but can not exclusively explain the prompt increases of temperature up to $39^{\circ} \mathrm{C}$ between 8.00 p.m. and 10.00 p.m.

In MS, most patients inject IFN- $\beta$ for better convenience and in order to oversleep possible side effects usually in the evening. Although our study provides a possible mechanism for IFN- $\beta$ induced side effects, we cannot conclude that the same mechanism accounts for patients with persistent FLS despite premedication with NSAID's. Should this be the case however, a reasonable consideration for such patients would be to alter the time point of injection to the morning. Another possibility would be an additional low dose oral glucocorticoid use at the onset of IFN- $\beta$ therapy as it has already been suggested by MartinezCaceres [16].

Plasma IL-10 concentrations also exhibited diurnal variations with higher baseline levels in the morning and a higher rise after IFN- $\beta$ injection at 8.00 a.m. These variations might be influenced by a circadian rhythmometry of IL-10 and/or by diurnal cortisol rhythm $[17,18]$. Increases of IL-10 during IFN- $\beta$ treatment have been observed in several studies and are attributed to the beneficial effects of IFN- $\beta$ therapy $[19,20]$. Plasma TNF- $\alpha$ levels were not affected by IFN- $\beta$ injection in this study. This might be due to the different dosage and route of administration (IFN $22 \mu \mathrm{g}$ s.c. versus $30 \mu \mathrm{g}$ i.m.) in comparison to the former studies, which showed a significant increase of TNF- $\alpha$ levels $8 \mathrm{~h}$ after IFN- $\beta$ injection $[4,5]$. Interestingly, we found diurnal variations of plasma TNF- $\alpha$ levels, which should be kept in mind for future studies when measuring this cytokine.

All acute responses to the initial IFN- $\beta$ injection adapted after 6 months of continuous treatment with IFN- $\beta$, while diurnal variations of temperature, plasma hormone and cytokine concentrations were still present. The reduced rise in temperature and IL- 6 after IFN- $\beta$ in the follow up investigation was associated with clearly less clinical side effects and is in line with the well known reduction of side effects in MS patients during the first months of IFN- $\beta$ therapy. IL-10 concentrations were higher in the follow up investigation in group A compared to group B and sTNF-RII levels at baseline were significantly elevated only in group A after continuous IFN- $\beta$ therapy. This point to differential long term effects on immunological parameters depending on the time of injection. Elevations of sTNF-RII during IFN- $\beta$ therapy have been reported by Laske et al. and Rieckmann et al. who found that patients with higher sTNF-RII concentrations during IFN- $\beta$ therapy exhibited less MRI disease activity thus possibly accounting to the beneficial effects of IFN- $\beta$ therapy 
$[21,22]$. On the other side, anti-TNF therapy with a recombinant TNF-receptor fusion protein, showed no beneficial effects but worsening in studies with MS patients [23].

So far dosing time for immunomodulatory therapies, is not an issue in MS but in other diseases such as cancer, it is already taken into consideration concerning effectiveness and toxicity of immunomodulatory and immunosuppressive drugs [24,25]. Further studies are necessary with bigger study populations and long term observations to address this question for MS patients during IFN- $\beta$ therapy.

In conclusion our results demonstrate that IL-6 is directly involved in the mediation of side effects after IFN- $\beta$ administration in MS patients and dosing time influenced acute changes of plasma hormone and cytokine concentrations. Time of injection was also related to the tolerability of IFN- $\beta$ therapy and might affect the efficacy of IFN- $\beta$ therapy, but the latter warrants further investigations with bigger study populations. Our results support the recommended method of increasing the IFN- $\beta$ dosage over several weeks to the maximum dosage when starting therapy in MS patients in order to slowly adapt the system to the IFN- $\beta$ challenge. Importantly the role of circadian rhythms should be taken into consideration when determining immunological parameters and/or performing immunomodulatory therapies.

\section{Acknowledgements}

We would like to thank Heike Staufer for continuous technical support.

This work was partially supported by an educational grant from Serono GmbH, Unterschleißheim, Germany.

\section{References}

1. PRISMS (Prevention of Relapses and Disability by Interferon beta-1a Subcutaneously in Multiple Sclerosis) Study Group. Randomised double-blind placebo-controlled study of interferon beta-1a in relapsing/ remitting multiple sclerosis. Lancet 1998; 52: 1498-504.

2. The IFN $\beta$ Multiple sclerosis Study Group. Interferon beta- $1 \mathrm{~b}$ is effective in relapsing-remitting multiple sclerosis. Clinical results of a multicenter, randomized, doubleblind, placebo-controlled trial. Neurology 1993; 43: 655-61.

3. Neilley LK, Goodin DS, Goodkin DE, Hauser SL. Side effect profile of interferon beta- $1 \mathrm{~b}$ in multiple sclerosis: results of an open label trial. Neurology 1996; 46: 552-54.

4. Goebel MU, Baase J, Pithan V, Exto MS, Saller B, Schedlowski $\mathbf{M}$ et al. Acute interferon beta-1b administration alters hypothalamic-pituitary-adrenal axis activity, plasma cytokines and leukocyte distribution in healthy subjects. Psychoneuroendocrinology 2002; 27: 881-92.
5. Kümpfel T, Then Bergh F, Pollmächer T, Holsboer F, Trenkwalder C. Acute effects of interferon beta-1a on plasma cytokine levels in patients with MS. Neurology 2000; 55: 1231-33.

6. Ohno Y, Fujimoto $M$, Nishimura A, Aoki $\mathbf{N}$. Change of peripheral levels of pituitary hormones and cytokines after injection of interferon (IFN)- $\beta$ in patients with chronic hepatitis C. J Clin Endocrinol Metabol 1998; 83: 3681-87.

7. Petrovsky N, McNair P, Harrison LC. Diurnal rhythms of proinflammatory cytokines: Regulation by plasma cortisol and therapeutic implications. Cytokine 1998; 10: 307-12.

8. Mormont MC, Levi F. Cancer chronotherapy: principles, applications, and perspectives. Cancer 2003; 97: $155-69$.

9. Ozaydin M, Dede O, Dogan A, Aslan MS, Altinbas A, Ozturk M et al. Effects of morning versus evening intake of atorvastatin on major cardiac event and restenosis in patients undergoing first elecvtive percutaneous coronary intervention. Am J Cardiol 2006; 97: 44-47.

10. Cutolo M, Sulli A, Pizzorni C, Secchi ME, Soldano S, Seriolo B et al. Circadian rhytms: glucocorticoids and arthritis. Ann N Y Acad Sci 2006; 1069: 289-99.

11. McDonald WI, Compston A, Edan G, Goodkin D, Hartung HP, Lublin FD et al. Recommended diagnostic criteria for multiple sclerosis: guidelines from the International Panel on the diagnosis of multiple sclerosis. Ann Neurol 2001; 50: 121-27.

12. Montalban $\mathbf{X}$, Duran $\mathbf{I}$, Rio J, Saez-Torres $\mathbf{I}$, Tintore M, Martinez-Caceres EM. Can we predict flu-like symptoms in patients with multiple sclerosis treated with interferon-beta? J Neurol 2000; 247: 259-62.

13. Nicoletti F, Marco $R$, Patti $F$, Zaccone $P$, L'Episcopo MR, Reggio E et al. Short-term treatment of relapsing remitting multiple sclerosis patients with interferon (IFN)-beta1B transiently increases the blood levels of interleukin (IL)-6, IL-10 and IFN- $\gamma$ without significantly modifying those of IL-1 $\beta$, IL-2, IL-4 and tumor necrosis factor- $\alpha$. Cytokine 2000; 12: 682-87.

14. Evans A, Main E, Zier K, Ikegaki N, Tartaglione Mkennett R, Lampson L. The effects of gamma interferon on natural killer and tumor cells of children with neuroblastoma. A preleminary report. Cancer 1989; 64: 1383-87.

15. Pollmächer T, Mullington J, Korth C, Schrieber W, Hermann D, Orth A et al. Diurnal variations in the human host response to endotoxin. J Infect Dis 1996; 174: $1040-45$.

16. Martinez-Caceres EM, Rio J, Barrau M, Duran BS, Borras BS, Tintore $\mathbf{M}$ et al. Amelioration of flulike symptoms at the onset of interferon beta-1b therapy in multiple sclerosis by low-dose oral steroids is related to a decrease in interleukin-6 induction. Ann Neurol 1998; 4: $682-85$

17. Young MR, Metthews JP, Kanabrocki EL, Sothern RB, Roitman-Johnson B, Scheving LE. Circadian rhythmometry of serum interleukin-2, interleukin-10, tumor necrosis factor alpha and granulocyte.macrophage colony-stimulating factor in men. Chronobiol Int 1995; 12: $19-27$.

18. Petrovsky N. Towards a unified model of neuroendocrineimmune interaction. Immunol and Cell Biol 2001; 79: $350-57$.

19. Ersoy E, Kus C, Sener U, Coker I, Zorlu, Y. The effects of interferon-beta on interleukin-10 in multiple sclerosis patients. Eur J Neurol 2005; 12: 208-11.

20. Rep MH, Schrijver HM, van Lopik T, Hintzen RQ, Roos MT, Ader HJ et al. Interferon (IFN)-beta treatment 
enhances CD95 and interleukin 10 expression but reduces interferon-gamma producing $\mathrm{T}$ cells in MS patients. J Neuroimmunol 1999; 96: 92-100.

21. Laske C, Oschmann P, Tofighi J, Kuehne SB, Diehl H, Bregenzer $\mathbf{T}$ et al. Induction of sTNF-R1 and sTNF-R2 by interferon beta-1b in correlation with clinical and MRI activity. Acta Neurol Scand 2001; 103: 105-13.

22. Rieckmann P, Kruse N, Nagelkerken L, Beckmann K, Miller D, Polmann C et al. Soluble vascular cell adhesion molecule (VCAM) is associated with treatment effects of interferon beta-1b in patients with secondary progressive multiple sclerosis. J Neurol 2005; 252: 526-33.

23. The Lenercept Multiple Sclerosis Study Group and the University of British Columbia MS/MRI Analysis Group. TNF neutralization in MS: results of a randomized, placebo-controlled multicenter study. Neurology 1999; 53: 457-65.

24. Mormont MC, Levi F. Cancer chronotherapy: principles, applications, and perspectives. Cancer 2003; 97: 155-69.

25. Ohdo S. Changes in toxicity and effectiveness with timing of drug administration: implications for drug safety. Drug Saf 2003; 26: 999-1010. 